\title{
Communication among health care teams: a major challenge for the improvement of patient safety
}

Advances in patient safety have progressed slowly in spite of efforts made in many countries. There are many hurdles in the path towards achieving changes that lead to a new medical culture where the priority is providing safe care to patients. Such obstacles include a scarce commitment from leaders, an inadequate approach to effectively evaluate factors that may harm patients, and the incorrect analysis of the reasons for errors. Only continuous and orderly work with a strong institutional support will help to mitigate adverse events. The development of skills and commitment from a small group of professionals members of hospital committees are no longer enough, and commitment is required from all leaders of medical institutions.

Different strategies include changes in health care professional training, participation of patients in their own care, transparency, coordinated care, and professionals with a "moral" motivation. The implication behind these requirements is that our attitude makes us responsible for preventing harm in patients, which constitutes an inherent ethical mandate. As doctors, we are not obliged to heal people, but we are obliged to cause no harm at all.

In addition, the obvious difficulties in progress lie in health care itself, which has traditionally been based on doctors' capability and autonomy, an incorrect approach which cannot be easily modified. Working alone, with a scarce and ineffective communication within a team and among the medical institution teams, is one of the most dire obstacles to provide safe care.

A team can be defined as "two or more people with specific roles, who perform independent tasks, are capable of adapting, and share a common objective." These characteristics are not enough to warrant that a team will function effectively because our work entails more than performing tasks and can be defined as "a set of interrelated knowledge, skills and attitudes enabling a coordinated and efficient performance" (Baker, et al, 2006). Only teams with such qualities will be able to share what they wish to attain.

Communication is a critical aspect among human beings and should be an effective part of all activities performed by individuals. Not surprisingly, an adequate communication is considered one of the core factors for maintaining safety and quality among work teams which perform complex activities. Long time ago, risk industries started to address adequate communication so as to increase their security to the maximum. Commercial aviation is probably the prime example with their marked accident reduction and becoming an industry that plans even the tiniest detail taking into account that people will inevitably fail and therefore, systems should prevent mistakes, or detect and correct them quickly.

Clearly, without effective communication, even competent people could be part of an incompetent team, as evidenced by the tragic explosion in the Chernobyl nuclear power station. The subsequent investigation on the causes of the accident revealed that the main reasons included inadequate or nearly inexistent communication among key teams.

Advances towards an adequate communication among teams in medicine have not yet reached a noteworthy magnitude, and unfortunately communication among professionals and with the administrative staff continues to be inefficient. For this reason, in spite of the great efforts made to improve patient safety, advances are still limited.

Undoubtedly, ineffective team work leads to patient harm. A review of more than 3500 sentinel events (resulting in death or serious injuries) conducted by The Joint Commission in the United States (2004) determined that $60 \%$ of deaths and $50 \%$ of serious injuries were caused by failure in team communication.

In such critical situation, a favorable aspect can be seen in the first attempts to investigate team communication and patient safety taking place within the difficult venture of medicine towards a cultural shift in the approach to medical errors.

Still, the more complicated aspects of communication have not been explored, and research cannot be excessively simple because this is an extremely complex process. More attention has been recently paid to the technical knowledge of human communications, which will help to establish an "effective communication" in medical institutions. Here I will briefly address only some points so as to better define this complex issue.

Lingard L. (Healthcare Quarterly, April 2012) described the three aspects she considered 
promising to contribute to the communication process. They include: the meaning of silence, the uptake of communication innovations, and the phenomenon of intertextuality. Until recently, communication research was based on the presence of speech, what team members are saying to one another, or what they should be saying to one another, in the course of their clinical work. Silence has received little attention, even when the importance of such aspect is evident to anyone who has been a member of a health care team at work. Without a doubt, "there is much more being communicated in teams than simply what is being said. The relation among members is full of meaningful silences" (Lingard L.).

Initially, studies on silence have explored why team members may not "speak up" during or after dangerous clinical situations. Error reporting assessment has demonstrated that this was because of the inadequate institutional safety culture that has led professionals to remain silent for fear of reprisal. As a result, the reporting system is weakened and the most reported errors are those considered mild or moderate.

However, not all silence is problematic, and communication experts have recognized silence as a rich resource. For this to work, it should be acknowledged that there is a distinction between "being silent" and "being silenced": the former may entail an understanding that does not need to be voiced, the latter may entail a restriction. An example of this may be the preparation of a safety checklist for the operating room. Gardezi, et al, (2009) described three forms of silence in this practice: not sharing information, not responding to requests, and speaking quietly; and found that such behaviors could be indicative of people being defensive and that silence constitutes a strategy to avoid commitment. An analysis of surgical errors (Christian C, et al. Surgery, 2006) revealed that lack of communication was the major causative factor.

The second aspect, the uptake of communication innovations, refers to several strategies to improve communication in special situations, such as the already mentioned operating room checklist, transition processes (hospital admission, transfer between units and discharge), "handovers," "patient handoff" and change of nurse shift. These situations favor a high error rate caused by inadequate communication. Possible strategies to be implemented are too many to be described here, but they basically deal with detecting and correcting communication weaknesses (see article by Belziti, et al., page 119).

The third aspect, the phenomenon of intertextuality, intends to go beyond of how information is orally transmitted among team members and studies how communication practices affect one another. Intertextuality has been used in other disciplines to better understand what is done and is really helpful to detect any shortcoming. It is widely known that in the medical field, information can get distorted as it is conveyed from one person to another in the context of an inadequate communication. The greatest risk is that such distortion can go unnoticed, therefore creating a fertile ground for errors to occur. An adequate and continuous assessment of team communication systems is mandatory for safety improvement. This will allow us to get to know the impact of communication practice on error occurrence and detect failures so as to avoid any potential "domino effect" leading to even more errors.

To conclude, I would like to highlight that physicians are involved in most communication processes, and there is no question that in those situations we still have the power, even though this may be detrimental. For this reason, no advance can be achieved if we, physicians, do not take the stand to develop and promote adequate communication among members of the teams we are part of. Our commitment to this challenge is essential, and we should bear in mind that no substantial change can be made in clinical care safety without our full participation. Only then we will be able to prevent or mitigate patient harm.

José M. Ceriani Cernadas Editor

http:/ /dx.doi.org/10.5546/aap.2014.eng.114 